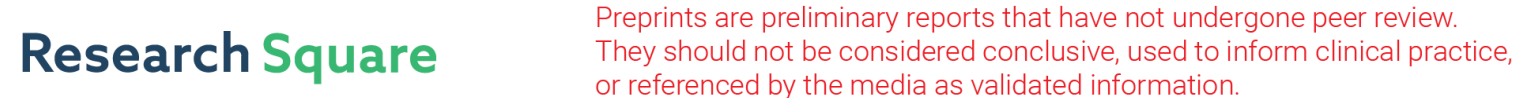 \\ Mathematical Model For Forwarding Packets In Communication Network
}

Samaa Adel Ibrahim Hussein ( $\sim$ samaaadel655@gmail.com )

Mansoura University

Fayez Wanis Zaki

Mansoura University

Mohammed Ashour

Mansoura University

\section{Research Article}

Keywords: SD-WAN, Phase type distribution, hyper- exponential disruption, hypo-exponential disruption, Matrix-Geometric

Posted Date: December 6th, 2021

DOI: https://doi.org/10.21203/rs.3.rs-1118104/v1

License: (c) (i) This work is licensed under a Creative Commons Attribution 4.0 International License. Read Full License

Version of Record: A version of this preprint was published at IET Networks on February 16th, 2022. See the published version at https://doi.org/10.1049/ntw2.12035. 


\title{
Mathematical Model for forwarding packets in communication network
}

\author{
Samaa Adel Ibrahim Hussein ${ }^{1, *}$, Fayez Wanis Zaki ${ }^{1}$, and Mohammed Ashour ${ }^{1}$ \\ ${ }^{1}$ Electronics and Communications Eng. Department Faculty of Engineering, Mansoura University- Egypt \\ *corresponding samaaadel655@gmail.com
}

\begin{abstract}
In recent years, SDN technology has been applied to several networks such as wide area network (WAN). IT provides many benefits, such as: enhancing data transfer, promoting Application performance and reducing deployment costs. Software Defined-WAN networks lack studies and references. This paper introduced a system for SD-WAN network using PH/PH/C queues. It concentrates on the study of algebraic estimates the probability distribution of the system states. The MatrixGeometric solution procedure of a phase type distribution queue with first-come first-served discipline is used.
\end{abstract}

Keywords-SD-WAN, Phase type distribution, hyper- exponential disruption, hypo-exponential disruption, Matrix-Geometric.

\section{Introduction}

In the modern internet age, many technologies should be developed to make the use of network resources and information exchange easier and faster ${ }^{1}$ Despite the various techniques that have been proposed to improve the WAN network, it faces some challenges such as: long distances, and multiple operators; which results in network congestion, packet delay changes, packet loss, together with service outages ${ }^{2}$. In addition to digital electronic wars, which are the fiercest and most dangerous wars for countries ${ }^{3}$. To overcome these limitations, the paper introduces some technologies of the $5 \mathrm{G}$, which is characterized by being more flexible, expendable and confidential in Software Defined wide-area networking research and technology ${ }^{4}$. Hence, SD-WAN considers addresses the current challenges of traditional networks. This new solution for network linking lowers operational costs, improves network resource utilization, and facilitates data deployment in multiple geographies. A software-defined WAN (SD-WAN) facilitates the management and control process through controlling from one central point through the separation of control layer from the data layer and providing better ways to connect many branches to each other in the main office. Also SD-WAN provides security, load balancing over multiple links, as well as easing of deployment and management incredibly ${ }^{5}$. Some literature and researches have focused on the study and application of Queue Modelling on SD-WAN networks to reduce waiting, such as: Wolthusen ${ }^{6}$ a model has been considered a queuing network for software defined industrial control networks. This model considers the strong periodic patterns of the network traffic in the data plane and derive the performance measures for the analytical model. Muhizi, et $\mathrm{al}^{7}$, as repeated a model an Openflow-SDN based on network visualization and performance evaluation model using $\mathrm{M} / \mathrm{H}_{2} / 1$ model. Goto, et $\mathrm{al}^{8}$, Proposed a model of the SDN network, with its accurate analysis of the model. Rapheal ${ }^{9}$, presented a study to find out the impact of QoS of SDN networks depending on the openflow switches. Zhao, et al ${ }^{10}$, he reported a model SD-WAN network based on a M/M/n queue through the messages-in and messages-out packet of controllers. Moreover, he studied properties and performance of OpenFlow switches as a model $\mathrm{M} / \mathrm{G} / 1$. Yang, et $\mathrm{al}^{11}$, proposed an accurate model of the SD-WAN network control system depending on the arrival process, as well as the optimal number of control units in the SD-WAN network.

\section{PROPOSED TOPOLOGY SD-WAN NETWORK}

Case study: As shown in Fig 1. SD-WAN in the proposed solution is broken up into four planes: data, control, management and orchestration. The proposed SD-WAN network can be described as containing a group of remote devices as known (vEdge router) which are located in the data plan. This paper focuses on Starting the process of connecting to the SD-WAN controllers and how to forwarding packet between vEdge routers. The vEdge router in all branches will communicate with orchestration plane to get services such as: update of operating system, and initial configuration. In addition to the information of the vBond, which sends all the communication information own by the other network components after the success of the authentication and authorization process. Finally management plan sends all the policies and configuration to the network as a whole. Noting that policies are created in the Management plan and are pushed via NETCONF either to vSmart controllers (centralized policies) or directly to vEdges (localized policies), (phase-1), as shown in Fig 2. 


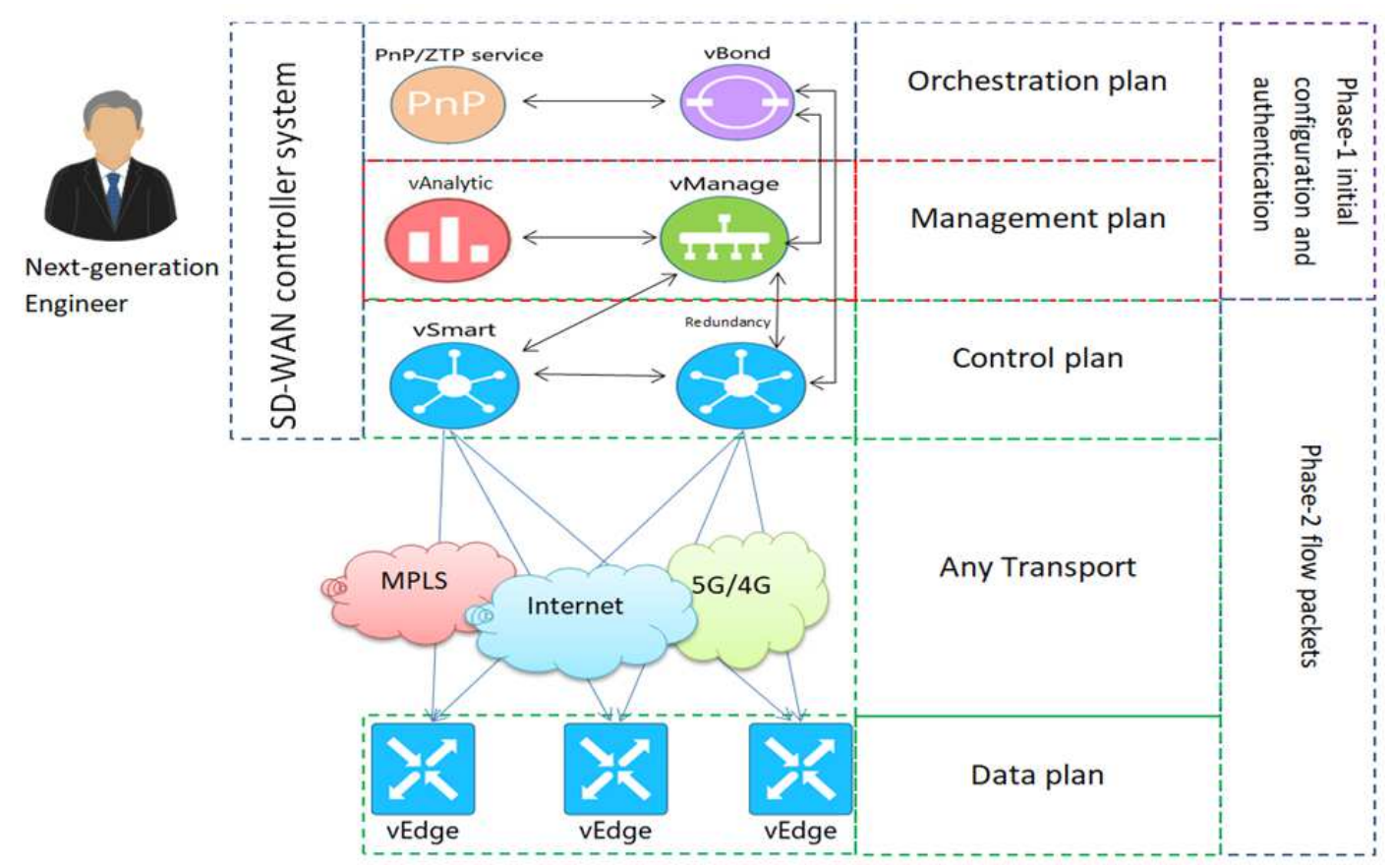

Figure 1. SD-WAN network

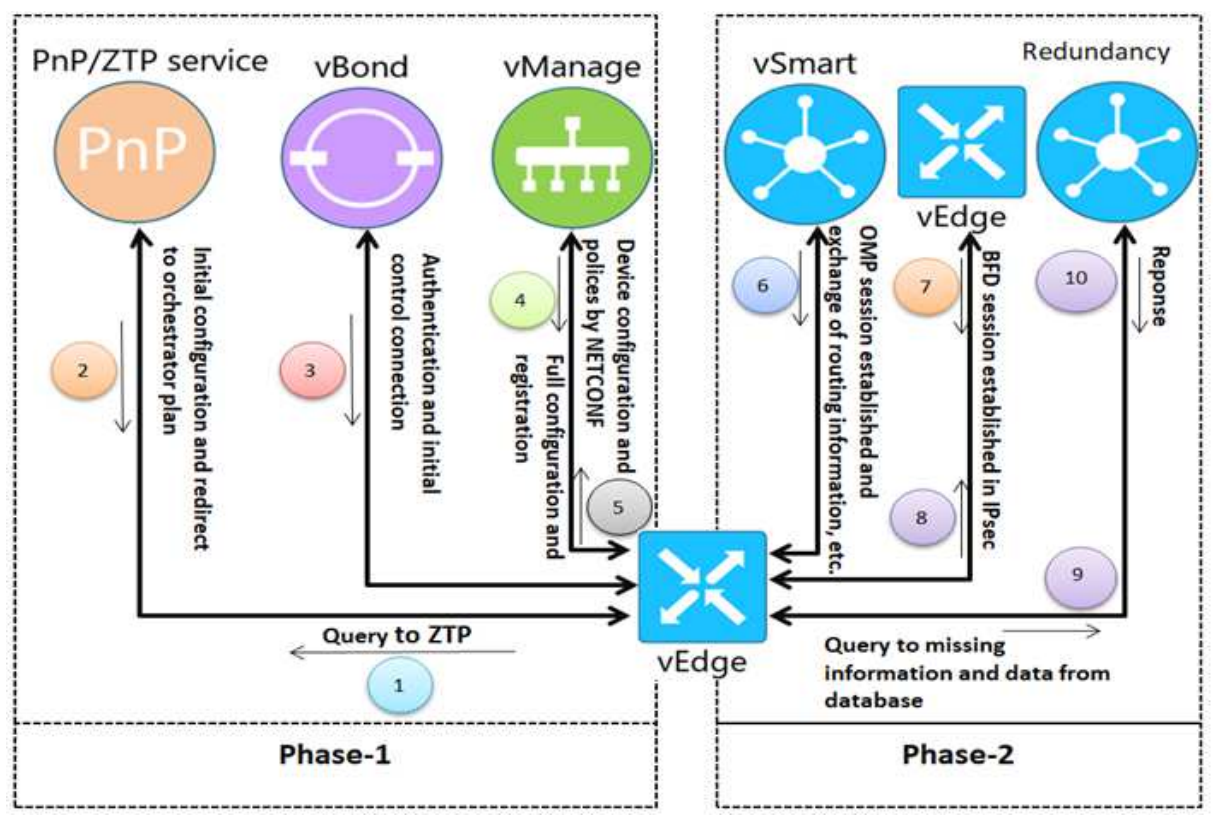

Figure 2. SD-WAN vEdge router connections

After the success of the first phase, vEdge router will communicate to the Control Plane to forward packets according to their routing table entries based on the decisions taken the SD-WAN controller system and interchange forwarding information with the SD-WAN controllers over the Overlay Management Protocol (OMP). The vSmart controller receives all routing and topology information from every vEdge router, calculates the best paths based on the policies, and then re-advertises the results to all other vEdge routers but never participate in the data-plane (packet forwarding). The operations of forwarding in packet vEdge router are arranged as shown in: Fig 4. 


\section{PROPOSED QUEUING MODEL FOR SD-WAN NETWORK}

$\mathrm{The} \mathrm{Ph} / \mathrm{Ph} / \mathrm{C}$ queue model consists of two processes as following: one is the arrival process model by Hyper-exponential Distribution with probability $\alpha_{i}, \sum_{i=1}^{k} \alpha_{i}=1$ and rates are given by: $\lambda_{1}, \lambda_{2}, \lambda_{3}$ and the other is a service process model by Hypo-exponential Distribution with two-stage hypo-exponential distribution for the service process with-its parameter $\mu_{1}, \mu_{2}$ as shown in Fig 3.

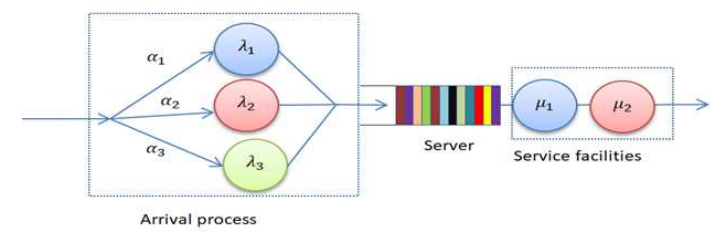

Figure 3. $\mathrm{Ph} / \mathrm{Ph} / \mathrm{C}$ queue with hyper-exponential and hypo-exponential

\subsection{Queuing model of SD-WAN controller System}

With SD-WAN under its management, $\mathrm{N}$ of the vEdge routers are supposed to be the packet flows with arrival rate: $\left(\lambda_{1}, \lambda_{2}, \ldots\right.$, $\left.\lambda_{i}\right)$. Subject to Phase-Type distribution with probability : $\left(\alpha_{1}, \alpha_{2}, \ldots, \alpha_{i}\right)$, the packet gets service at a rate $\mu_{i}$ with probability $\alpha_{i}$ and leave the controller. Only one packet enters the controller and then comes out after serving it. Fig 3 shows arrival Phases which cannot be active the same time ${ }^{10}$ where:

$$
\lambda_{c}=\sum_{i=1}^{N}\left(1-\alpha_{i}\right) \lambda_{i}
$$

$\lambda_{c}$ is the volume of arrivals for unit time (packet/ms), arrival rate $\lambda_{i}, \mathrm{i} \geq 1$ and arrival rate are applied to the hyper-exponential distribution by $F(.)^{7}$. The probability density function of the arrival packet $\mathrm{X}$ can be given by:

$$
\begin{aligned}
& f_{X}(x)=\alpha_{1} \lambda_{1} e^{-\lambda_{1} x}+\alpha_{2} \lambda_{2} e^{-\lambda_{2} x}+\alpha_{3} \lambda_{3} e^{-\lambda_{3} x} \\
& f_{X}(x)= \begin{cases}\sum_{i=1}^{k} \alpha_{i} \lambda_{i} e^{-\lambda_{i} x} & x \geq 0 \\
0 & x \text { otherwise }\end{cases}
\end{aligned}
$$

while the cumulative distribution function can be expressed as:

$$
\begin{aligned}
& F_{X}(x)=\alpha_{1}\left(1-e^{-\lambda_{1} x}\right)+\alpha_{2}\left(1-e^{-\lambda_{2} x}\right)+\alpha_{3}\left(1-e^{-\lambda_{3} x}\right) \\
& F_{X}(x)= \begin{cases}1-\sum_{i=1}^{k} \alpha_{i} e^{-\lambda_{i} x} & x \geq 0 \\
0 & x \text { otherwise }\end{cases}
\end{aligned}
$$

where $\sum \alpha_{i}=1, \alpha_{i}$ is the probability that $\mathrm{X}$ will take on the form of the hyper-exponential distribution with rate $\lambda_{i}$. The first and second moments may be expressed as ${ }^{12}$ :

$$
\begin{aligned}
& E[X]=\frac{\alpha_{1}}{\lambda_{1}}+\frac{\alpha_{2}}{\lambda_{2}}+\frac{\alpha_{3}}{\lambda_{3}} \\
& E\left[X^{2}\right]=\frac{2 \alpha_{1}}{\left(\lambda_{1}\right)^{2}}+\frac{2 \alpha_{2}}{\left(\lambda_{2}\right)^{2}}+\frac{2 \alpha_{3}}{\left(\lambda_{3}\right)^{2}}
\end{aligned}
$$

The distribution $\mathrm{F}($.) on the interval $[0, \infty)$ can be Phase Type distribution as its representation $(\eta$, T ) where $\eta$ is a row vector of dimension $\mathrm{m}$ denotes Probability that phase is taken and $\mathrm{T}$ is a square matrix of dimension $\mathrm{m}$ that denote arrival processes ${ }^{13}$. The transition matrix of arrival rate $\grave{T}$ is given as follows:

$$
\grave{T}=\left[\begin{array}{ccc|c}
-\lambda_{1} & 0 & 0 & \lambda_{1} \\
0 & -\lambda_{2} & 0 & \lambda_{2} \\
0 & 0 & -\lambda_{3} & \lambda_{3} \\
\hline 0 & 0 & 0 & 0
\end{array}\right]
$$


The generator $\grave{T}$ of a Markov chain can be written as:

$$
\grave{T}=\left[\begin{array}{ll}
T & T^{0} \\
0 & 0
\end{array}\right]
$$

and initial probability distribution $\grave{\eta}$ is given by:

$$
\begin{gathered}
\grave{\eta}=\left(\begin{array}{cccc}
\alpha_{1} & \alpha_{2} & \alpha_{3} & 0
\end{array}\right) \\
=\left(\begin{array}{ll}
\eta & 0
\end{array}\right)
\end{gathered}
$$

. Where u points a column vector with all elements equal to one, and the vector $T^{0}=-\mathrm{Tu}$. The coefficient of variation is:

$$
E\left[X^{2}\right]-(E[X])^{2}=C_{x}^{2}=\frac{E\left[X^{2}\right]-(E[X])^{2}}{(E[X])^{2}}-1 \geq 1 .
$$

\subsection{Queuing model of vEdge router (branch)}

Assuming that all packet switching process in vEdge routers are series of sequential k-phase exponential distribution. Each with its own rate $\mu_{i}$, the rate of the $\mathrm{i}^{\text {th }}$ distribution. Which follows a hypo-exponential distribution ${ }^{14}$. The service process distribution $H($.$) can be represented by the pair (\sigma, \mathrm{S})$ of dimension n, where: $\mathrm{S}$ is service process and $\sigma$ is initial probability. In this paper it is assumed that there are two phases of service inside SD-WAN controller.The transition rate matrix $=$ service processes $=\grave{S}$, thus we can write generator $\grave{S}$ of such a Markov chain?as:

$$
\begin{gathered}
\grave{S}=\left[\begin{array}{cc|c}
-\mu_{1} & \mu_{1} & 0 \\
0 & -\mu_{2} & \mu_{2} \\
\hline 0 & 0 & 0
\end{array}\right] \\
=\left(\begin{array}{cc}
S & S^{0} \\
0 & 0
\end{array}\right)
\end{gathered}
$$

as well as Initial probability distribution $\check{\sigma}$ as :

$$
\begin{gathered}
=\left(\begin{array}{ll|l}
1 & 0 & 0
\end{array}\right) \\
=\left(\begin{array}{ll}
\sigma & 0
\end{array}\right)
\end{gathered}
$$

Note that any initial probability must be equal one, so that $\eta \mathrm{u}=1$ and $\sigma \mathrm{u}=1$, where $\mathrm{u}$ is a unity column vector. Whereas a probability distribution $\mathrm{H}(.)^{15}$ on the interval $[0, \infty)$ is of phase type if it can arise as the absorption time distribution of $(\mathrm{k}+1)$ state Markov chain with $\mathrm{m}$ transient states $1,2, \ldots, \mathrm{k}$ and the absorbing state is 0 . The probability density function (pdf) as can be given by:

$$
\begin{aligned}
& F_{X}(x)= \begin{cases}\frac{\mu_{1} \mu_{2}}{\mu_{1}-\mu_{2}} e^{-\mu_{1} x} e^{-\mu_{2} x} & x \geq 0 \\
0 & \text { otherwise }\end{cases} \\
& f(x)=\sum_{i=1}^{k} \alpha_{i} \mu_{i} e^{\left(-\mu_{i} x\right)}, \text { where } \alpha_{i}=\left(\prod_{j=1, j \neq i}^{k} \frac{\mu_{i}}{\mu_{j}-\mu_{i}}\right)
\end{aligned}
$$

The cumulative distribution function is given by:

$$
F(x)=1-\frac{\mu_{2}}{\mu_{2}-\mu_{1}} e^{\left(-\mu_{1} x\right)}+\frac{\mu_{1}}{\mu_{2}-\mu_{1}} e^{\left(-\mu_{2} x\right)}, x \geq 1 .
$$

The expectation, variance, and squared coefficient of service process may be expressed as:

$$
\begin{aligned}
& E[X]=\frac{1}{\mu_{1}}+\frac{1}{\mu_{2}}, \operatorname{Var}[X]=\sigma^{2}=\frac{1}{\mu_{1}^{2}}+\frac{1}{\mu_{2}^{2}}, \\
& C_{x}^{2}=\frac{\mu_{1}^{2}+\mu_{2}^{2}}{\left(\mu_{1}+\mu_{2}\right)^{2}}
\end{aligned}
$$

The coefficients of variation are always $<1$. 


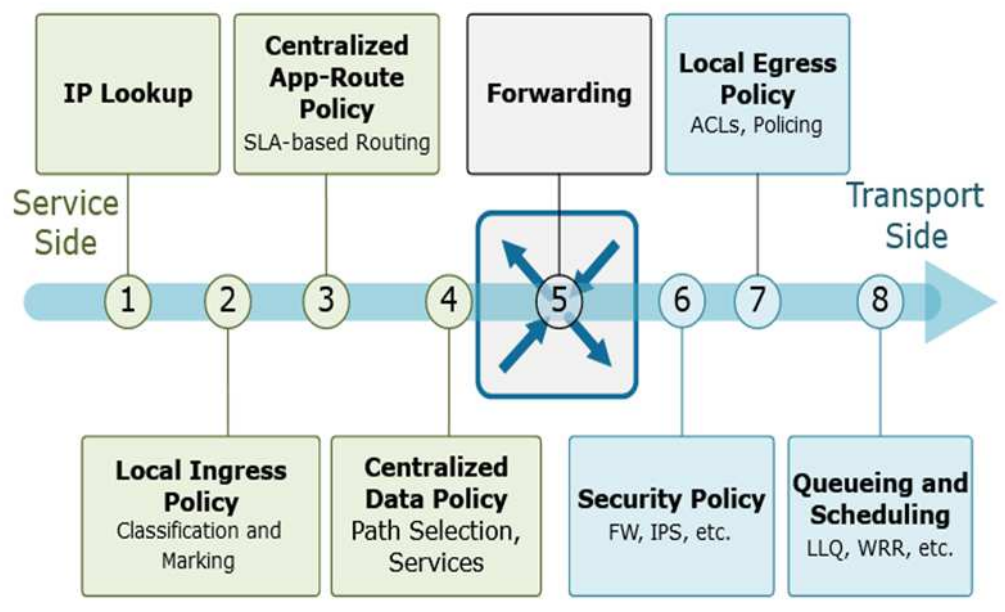

Figure 4. The Order Operations of an Edge router

\section{MATHEMATICAL ANALYSIS OF PACKET FORWARDING}

vEdge router analyses, the first step in the packet forwarding process is to perform a routing lookup on the destination IP of the packet in the routing table. If the match is successful, the configured action is performed usually where forwarded data to the next step, or through a specific port. Otherwise, the packets will be dropped or sent to a temporary store for processing or resent to the SD-WAN controller system. According to the policies set by the SD-WAN controller system , the action applied (by default it is reject).All packets that sent in the first and second Phase from the SD-WAN system to the vEdge routers are forwarded in a form of service queuing packets. Represented in two phases of service apply to it phase-type distribution and provided by a group of controllers as follows: The first Phase is the preparation, authentication and initial-configuration, it takes place in the orchestration plane, and it is served by two types of controllers :( Zero-Touch Provisioning (ZTP) server and vbond controller).The second Phase is the data flow between the ends of the network, forwarding packet and policies between network devices at the data plan. While the packets sent by the vEdge router are forwarded in a form of arrival queuing packets.as given below: The probability that the vEdge router is sending arrival packet to SD-WAN system asking for more information for the initial contact information, update operation system, upgrade or authentication process, probability that the vEdge router is sending arrival packet to SD-WAN system asking information about routing, forwarding data, update routing table or topology, and probability that the vEdge router is sending to arrival packet to SD-WAN system asking to Provide it with missing information and data from database which is in the redundancy controller.

\subsection{Mathematical analysis:}

There are four basic steps to a solving phase-type queuing systems using matrix-geometric approach as follows:

1. Building the sub-matrices: suppose that the phase arrival process is given as $r_{a}=r_{3}$ and services process given as $r_{s}=r_{2}$. The block sub-matrices $A_{0}, A_{1}, A_{2}, B_{00}, B_{10}, B_{01}$ can be constructed by applying matrix-geometric approach ${ }^{14}$ yields the following: $r_{a}=3$ and $r_{s}=2$ that is :

$$
\begin{gathered}
A_{0}=I_{r a} \otimes\left(s^{0} \cdot \sigma\right) \\
A_{1}=T \otimes I_{r s}+I_{r a} \otimes S=T \otimes I_{2}+I_{3} \otimes S \\
A_{2}=\left(T^{0} \cdot \eta\right) \otimes I_{2} \\
A=A_{1}+A_{2}+A_{3} \\
B_{00}=T \\
B_{01}=\left(T^{0} \cdot \eta\right) \otimes \sigma \\
B_{10}=I_{r a} \otimes S^{0}
\end{gathered}
$$

It was mentioned in section 3.2 that : $\mathrm{S}, S^{0}, \mathrm{~T}, T^{0}, \eta, \sigma$ and $I_{n}$ is the identity matrix of order $\mathrm{n}$, where $\mathrm{n}$ either $\left(r_{a}\right.$ or $\left.r_{s}\right)$, $A_{0}$ represented service completions at rate $\mu_{2}, A_{2}$ represented arrivals completions at rate $\alpha_{i} \lambda_{i}$ based on number of states, 
$A_{1}$ super-diagonal elements represented service completions at rate $\mu_{1}$, and the matrices $B_{i j}$ represent initial conditions. The transition rate matrix $(\mathrm{Q})$ given as:

$$
Q=\left[\begin{array}{cccccc}
B_{00} & B_{01} & 0 & 0 & \ldots & 0 \\
B_{10} & A_{1} & A_{2} & 0 & \vdots & 0 \\
0 & A_{0} & A_{1} & A_{2} & \ldots & 0 \\
0 & 0 & A_{0} & A_{1} & A_{2} & 0 \\
\ldots & \ddots & \ddots & 0 & 0 & \ldots \\
0 & 0 & \ldots & 0 & \vdots & \ldots
\end{array}\right]
$$

Since $\pi_{i}$ is vector refers to unique stationary probability distribution (steady-state distribution) can describe a continuoustime Markov chain as given by this equation:

$$
\pi Q=0, \text { with } \pi u=1
$$

From equation 10 the general case in $\mathrm{PH} / \mathrm{PH} / \mathrm{C}$ queue can be written as:

$$
\lambda \pi_{i-1}-(\lambda+\mu) \pi_{i}+\mu \pi_{i+1}
$$

by dividing the equation to $\mu$ to produce $\pi_{i+1}=\left(\frac{\lambda+\mu}{\mu}\right) \pi_{i^{-}}\left(\frac{\lambda}{\mu}\right) \pi_{i-1}=\left(\frac{\lambda}{\mu}\right) \pi_{i} \quad \mathrm{i}=1,2, \ldots$ knowing that the utilization factor denotes $\rho=\frac{\lambda}{\mu}$ hence the final equation of stationary probability may be written as:

$$
\pi_{i}=\left(\frac{\lambda}{\mu}\right)^{i} \pi_{0}=\rho^{i} \pi_{0}=\pi_{i-1} R,
$$

as geometrically

$$
p i_{i}=\pi_{1} R^{i-1}
$$

In equation 12 assuming that $\pi_{0}$ is known, hence evaluating the value of $\pi_{i}$ is easy to be obtained. In QBD process, the parameter $\rho$ will be defined a square matrix R of order $\mathrm{k} \times \mathrm{k}$ and $\pi_{i}$ will be a vector of order $\mathrm{K}$, where $\pi_{i}=\left(\pi_{0}, \pi_{1}, \pi_{2}\right.$ $\left., \ldots, \pi_{i}\right)$. Accordingly, equation 12 can be re-expressed as:

$$
\begin{gathered}
\pi_{0} B_{00}+\pi_{1} B_{10}=0, \\
\pi_{0} B_{01}+\pi_{1} A_{1}+\pi_{2} A_{0}=0, \\
\pi_{1} A_{2}+\pi_{2} A_{1}+\pi_{3} A_{0}=0, \\
\vdots \\
\pi_{i-1} A_{2}+\pi_{i} A_{1}+\pi_{i+1} A_{0}=0, \quad i=1,2, \ldots
\end{gathered}
$$

By substituting in equation 13 can be found $\mathrm{R}$ as follows:

$$
\begin{array}{r}
\pi_{1} R^{i-2} A_{2}+\pi_{1} R^{i-1} A_{1}+\pi_{1} R^{i} A_{0}=0 \\
\pi_{1} R^{i-2}\left(A_{2}+R A_{1}+R^{2} A_{0}\right)=0 \\
A_{2} A^{-1}+R+R^{2} A_{0} A_{1}^{-1}=0 \\
\\
\end{array}
$$

2. Form Neuts' R matrix from Equation 14is as follows:

$$
R_{K+1}=-V-R_{K}^{2} W .
$$

Where $\mathrm{V}=A_{2} A_{1}{ }^{-1}, \mathrm{~W}=A_{0} A_{1}^{-1}$, with initial value $R_{(0)}=0$, and $\mathrm{k}=1,2, \ldots$ 
3. Solve the boundary equations as follows: In the system as described previously in Fig. (3) given by Q matrix, the boundary equations are:

$\pi_{0}\left(\begin{array}{ll}B_{00} & A_{1}+R A_{0}\end{array}\right)=0$, solved for $\pi_{0}$ and thereafter used $\pi_{i}=\pi_{0} R^{i}, \mathrm{i}=1,2, \ldots$, to obtain the stationary probability vector as:

$$
\left(\begin{array}{ll}
\pi_{0} & \pi_{1}
\end{array}\right)\left(\begin{array}{cc}
B_{00} & B_{01} \\
B_{10} & A_{1}+R A_{0}
\end{array}\right)=0
$$

where $\pi_{i}=\pi_{1} R^{i-1}, \mathrm{i}=2,3, \ldots$ to provide a unique solution, let set $\pi_{0}=1$ as installed by :

$$
\begin{gathered}
1=\sum_{i=0}^{\infty} \pi_{i} \mathrm{u}= \\
\pi_{0} \mathrm{u}+\sum_{i=1}^{\infty} \pi_{1} R^{i-1} \mathrm{u}= \\
\pi_{0} \mathrm{u}+\sum_{i=0}^{\infty} \pi_{1} R^{i} \mathrm{u}=
\end{gathered}
$$

$$
\pi_{0} u+\pi_{1}(I-R)^{-1} u
$$

Let $\mathrm{A}=A_{0}+A_{1}+A_{2}$ and $\pi_{A} \mathrm{~A}=0$. The following condition ought to be held for a $\mathrm{QBD}$ process:

$$
\pi_{A} A_{2} u<\pi_{A} A_{0} u
$$

4. The successive components of the solution can be easily generated to build stationary probability vector by using Matlab Packages as illustrated in?

\subsection{System Characteristics and mathematical calculations:}

The stability condition for the proposed system? can be given by:

$$
\mathrm{E}[\mathrm{S}]<\mathrm{E}[\mathrm{A}]
$$

where

$$
E[S]=\frac{1}{\mu_{1}}+\frac{1}{\mu_{2}}
$$

and

$$
E[A]=\frac{\alpha_{1}}{\lambda_{1}}+\frac{\alpha_{2}}{\lambda_{2}}+\frac{\alpha_{3}}{\lambda_{3}}
$$

Where E[S]: mean service time, and E [A]: mean inter arrival time, the system is stable when traffic intensity $\rho<1$, it means that ratio average arrival $\rho=\frac{\lambda_{\text {mean }}}{C \mu_{\text {mean }}}$, $(\rho$ must be $<1)$.

\subsection{Performance measurements:}

- The probability that there are $\mathrm{k}$ packets existing within the queuing system can be expressed as:

$$
\begin{aligned}
& P_{k}=\left\|\pi_{k}\right\|_{1}=\left\|\pi_{1} R^{k-1}\right\|_{1} \\
& \quad \text { Prob }\{\mathrm{N} \geq \mathrm{k}\}=\sum_{j=k}^{\infty}\left\|\pi_{j}\right\|_{1}=\left\|\pi_{1} \sum_{j=k}^{\infty} R^{j-1}\right\|_{1} \\
& =\left\|\pi_{1} R^{k-1} \sum_{j=0}^{\infty} R^{j}\right\|_{1}=\left\|\pi_{1} R^{k-1}(I-R)^{-1}\right\|_{1}
\end{aligned}
$$

it can be argued that the probability of the system is not busy when it is at:

$$
P_{0}=\left\|\pi_{0}\right\|_{1}=1-P_{k}=1-\rho
$$


- The average number of packets in $\mathrm{Ph} / \mathrm{Ph} / \mathrm{C}$ queuing system is given by:

$$
\begin{gathered}
\mathrm{E}[\mathrm{V}]=\sum_{k=1}^{\infty} \mathrm{k}\left\|\pi_{k}\right\|_{1}=\sum_{k=1}^{\infty} \mathrm{k}\left\|\pi_{1} R^{k-1}\right\|_{1} \\
=\pi_{1} \sum_{k=1}^{\infty} \frac{d}{d R} R^{k} \|_{1}= \\
\left\|\pi_{1} \frac{d}{d R}\left(\sum_{k=1}^{\infty} R^{k}\right)\right\|_{1}=\left\|\pi_{1} \frac{d}{d R}\left((I-R)^{-1}-I\right)\right\|_{1} \\
=\left\|\pi_{1}(I-R)^{-2}\right\|_{1}
\end{gathered}
$$

- The packets average number waiting within the queue may be given by:

$$
E\left[V_{q}\right]=E[V]-\frac{\lambda}{\mu}
$$

- The average response time is expressed as:

$$
E[T]=\frac{E[V]}{\lambda} .
$$

- The average time of arrival packets waiting in system may be expressed as:

$$
E\left[W_{q}\right]=\frac{E\left[V_{q}\right]}{\lambda}
$$

- Mean queue length is defined as :

$$
L_{q}=E\left[V_{q}\right]=\sum_{k=1}^{\infty} K P_{k}-\sum_{k=1}^{\infty} P_{k}=L-\left(1-P_{0}\right)
$$

where : $L=L_{q}+L_{S}$ and $L_{q}=\rho L$ and $L=E[V]$, L denotes the total length of the queue, $L_{q}$ is the queue arrival length, $L_{S}$ denotes the queue service length, and $L_{s}=\sum_{k=1}^{\infty} K P_{k}$.

- The throughput of the queuing system is defined as the departure rate $X=\lambda$ if all arrived packets get their service and leave the SD-WAN controller system ${ }^{16}$. The properties of the queuing system are given in Table 1.

\subsection{Markov process and state transitions diagram}

The process can be visualized as a graph with the transition probabilities as illustrated in Fig 5 and Fig 6.

- The describe of state in network $(\mathrm{k}, \mathrm{a}, \mathrm{s})$ where: $\mathrm{k}$ denotes the number of packets in system, a denotes arrival phases of arriving packet and $\mathrm{s}$ denotes current phases of service ${ }^{17}$.

- Vertical transitions represent arrival rate as shown in Fig 5 in phase-1,the transition from state $(\mathrm{k}, 1, \mathrm{~s})$ for stating $(\mathrm{k}+1,1$, $\mathrm{s})$; at rate $\lambda_{1} \alpha_{1}$, phase 2 , the system shift from state $(\mathrm{k}, 1, \mathrm{~s})$ to state $(\mathrm{k}+1,2, \mathrm{~s})$ at rate $\lambda_{1} \alpha_{2}$, and phase-3 the system moves from state $(\mathrm{k}, 1, \mathrm{~s})$ to state $(\mathrm{k}+1,3, \mathrm{~s})$ at rate $\lambda_{1} \alpha_{3}$.

- Horizontal transitions represent service rate as shown in Fig 6 The transitions at rate $\mu_{1}$ move the system from states $(\mathrm{k}, \mathrm{a}, 1)$ to state $(\mathrm{k}, \mathrm{a}, 2)$; while $\mu_{2}$ move from the state $(\mathrm{k}, \mathrm{a}, 2)$ towards state $(\mathrm{k}-1, \mathrm{a}, 1)$, for $\mathrm{k}=1,2, \ldots$ 
Table 1. Properties of queuing system

\begin{tabular}{|l|l|l|}
\hline property & value & Explain \\
\hline $\begin{array}{l}\text { check } \\
\text { stability } \\
\text { of system }\end{array}$ & $\rho=0.0048 \%<1$ & System is stable \\
\hline $\begin{array}{l}\text { Queue } \\
\text { Notation }\end{array}$ & $\mathrm{PH} / \mathrm{PH} / 1$ & $\begin{array}{l}\text { Kendall notation for the } \\
\text { queue [Phase type disrup- } \\
\text { tion ] }\end{array}$ \\
\hline $\begin{array}{l}\text { Arrival } \\
\text { Rate }\end{array}$ & $0.588 \frac{\text { packet }}{m s}$ & $\begin{array}{l}\text { arrival rate for the queu- } \\
\text { ing system }\end{array}$ \\
\hline $\begin{array}{l}\text { Service } \\
\text { rate }\end{array}$ & $122 \frac{\text { packet }}{m s}$ & $\begin{array}{l}\text { Service rate for each } \\
\text { server }\end{array}$ \\
\hline $\begin{array}{l}\text { System } \\
\text { Capacity }\end{array}$ & $\infty$ & $\begin{array}{l}\text { maximum capacity of the } \\
\text { queuing system }\end{array}$ \\
\hline Throughput $X=\mathrm{C} \lambda=3.528 \times e^{9}$ & $\begin{array}{l}\text { departure rate for the } \\
\text { queuing system }\end{array}$ \\
\hline $\begin{array}{l}\text { Utilization } \\
\text { Factor }(\rho)\end{array}$ & $0.0048 \frac{\text { packet }}{m s}$ & $\begin{array}{l}\text { Fraction of time the } \\
\text { servers are busy }\end{array}$ \\
\hline
\end{tabular}

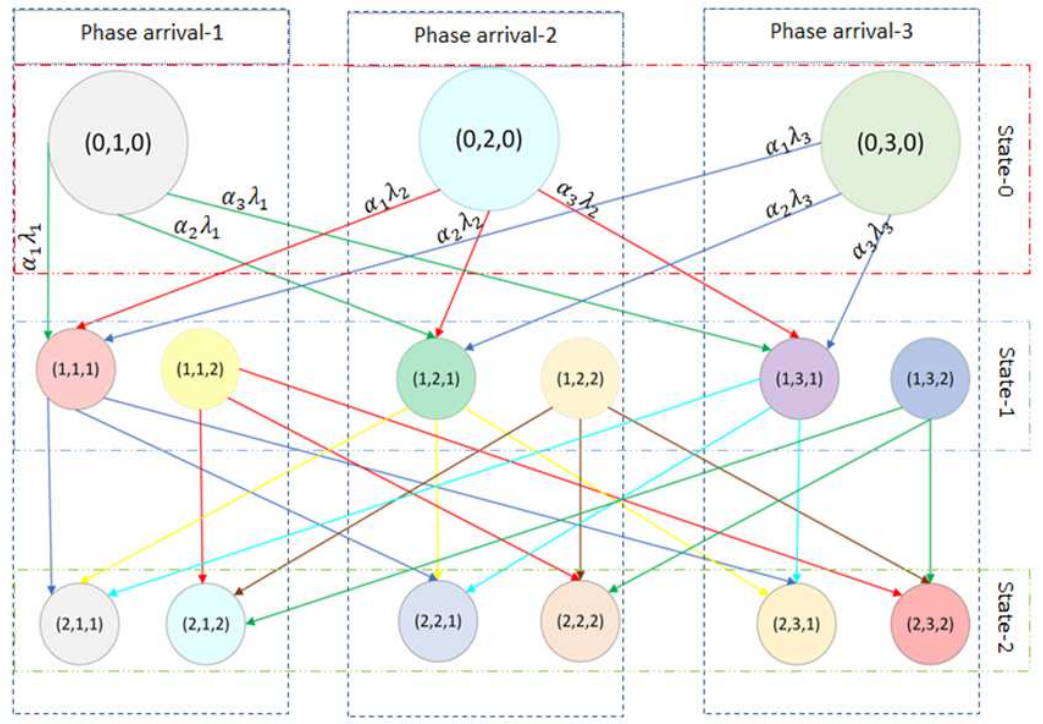

Figure 5. Transitions state arrival rate

\subsection{Simulation and results:}

Through the present paper, efficiency, effectiveness as well as stability of the proposed SD-WAN network topology are considered. In this section the performance measurement is studied by MATLAB simulate and the results are persecuted and discussed. It was resorted to studying the SD-WAN network to improve the performance of wide area networks and reduce lost packages, as more than one branch is connected to the main canter. There are many factors affecting network performance and the speed of data transmission between WAN branches such as: utilization factor (system load), arrival rate, system size, system time Queue size and Queue time. In the following, several results obtained from the previous study will be presented. The Fig 7 refers to the improvement of SD-WAN throughput compared to traditional networks, as the less the system utilization, the higher the throughput.

The second result as shown Fig 8 the delay in traditional networks is much greater than SD-WAN networks, as in the experiment of this paper the delay in SD-WAN networks is less than $(1.2 \mathrm{~ms})$ than traditional networks, and therefore the system response speed is less in traditional networks than SD-WAN networks.

Also, one of the most important results that have been reached is as shown in Fig 9 that the queuing time in traditional networks is greater than in SD-WAN. 


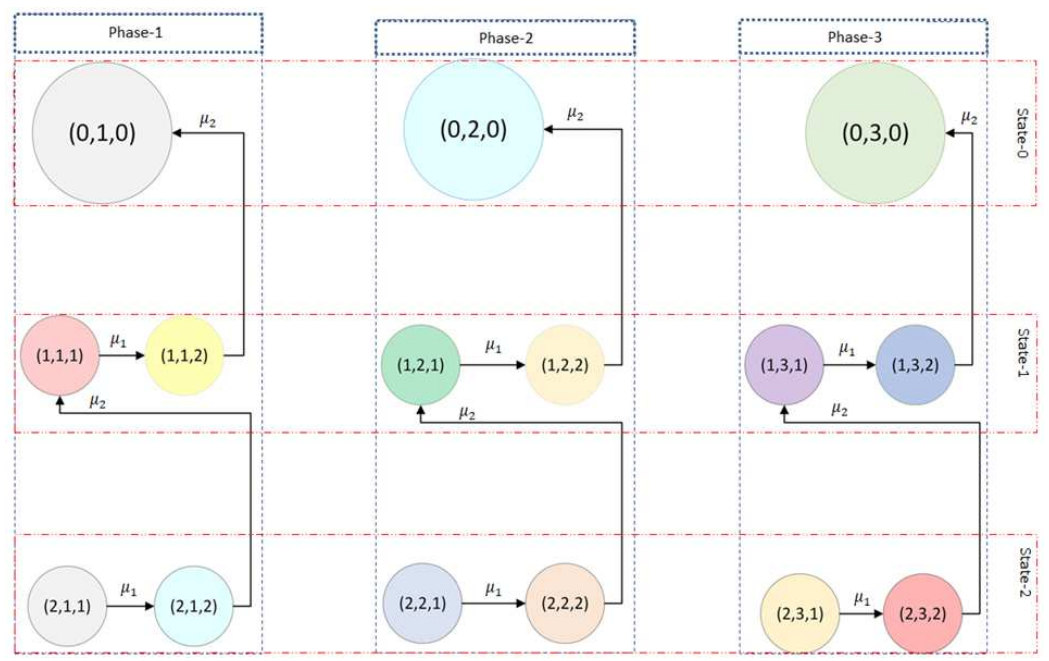

Figure 6. Transitions state service rate

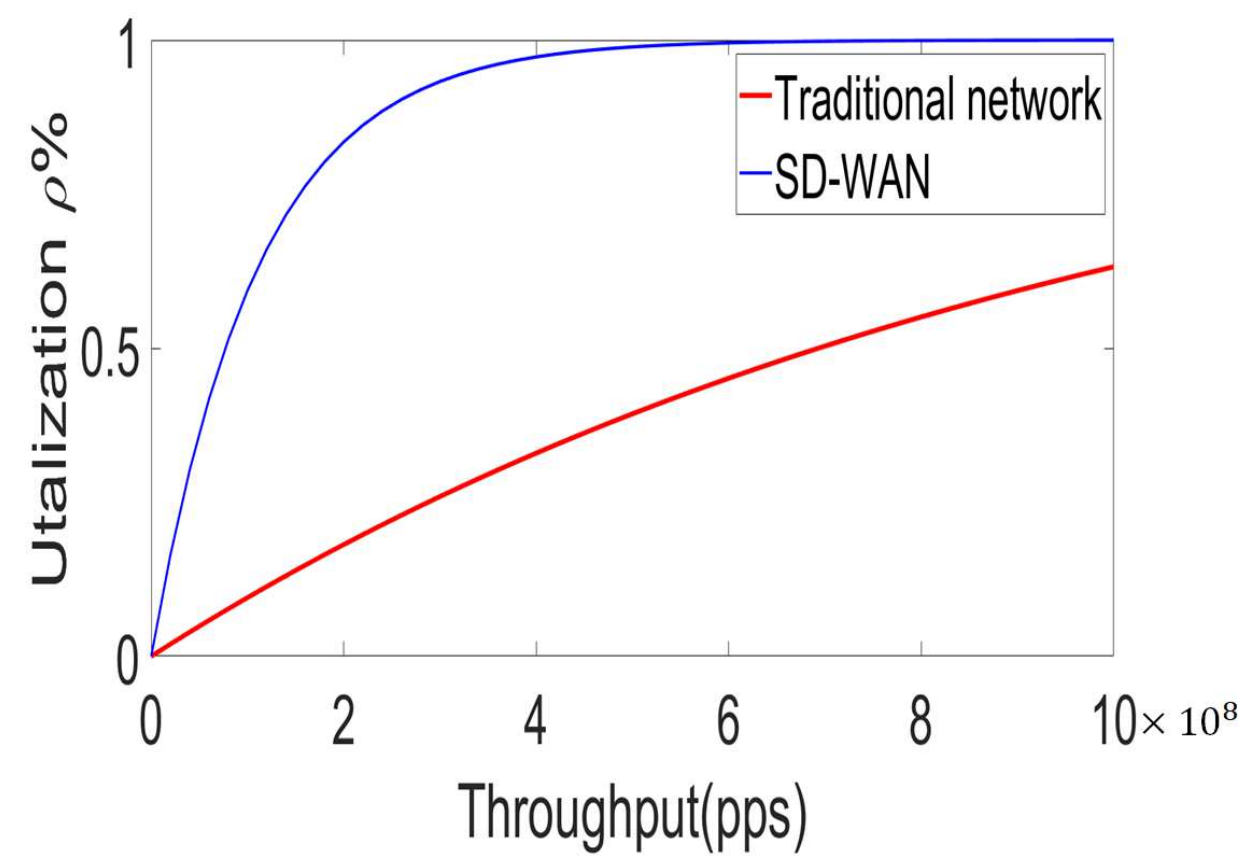

Figure 7. Difference between Throughput in SD-WAN and traditional networks

The number of arrival packet in traditional networks is large, and therefore the system utilization is high. Accordingly, the waiting packets will be large in traditional networks compared to the SD-WAN as Fig 10.

The Fig 11 shows that with the decrease in the utilization system, the service rate increases, and thus, the service time will be reduced with reduced arrival rate.

At the end the service rate in SD-WAN improved by a very significantly due to the separation of the control plan from the data plan, because to the number of operations decreased and the consumption of network resources as shown in Fig 12.

\section{Conclusion}

The SD-WAN network has some limitations that need to be taken into consideration, such as congestion in arrival packets, and this will lead to a delay in entering the packet to take the service, and thus a Queuing delay is the time a arrival packet waits in a queue until it can be serviced will occur in the network. This paper is motivated to propose an objective for modelling together with analysis systems for $\mathrm{PH} / \mathrm{PH} / \mathrm{C}$ queues aids in minimizing their problems, testing efficiency of the proposed SD-WAN 


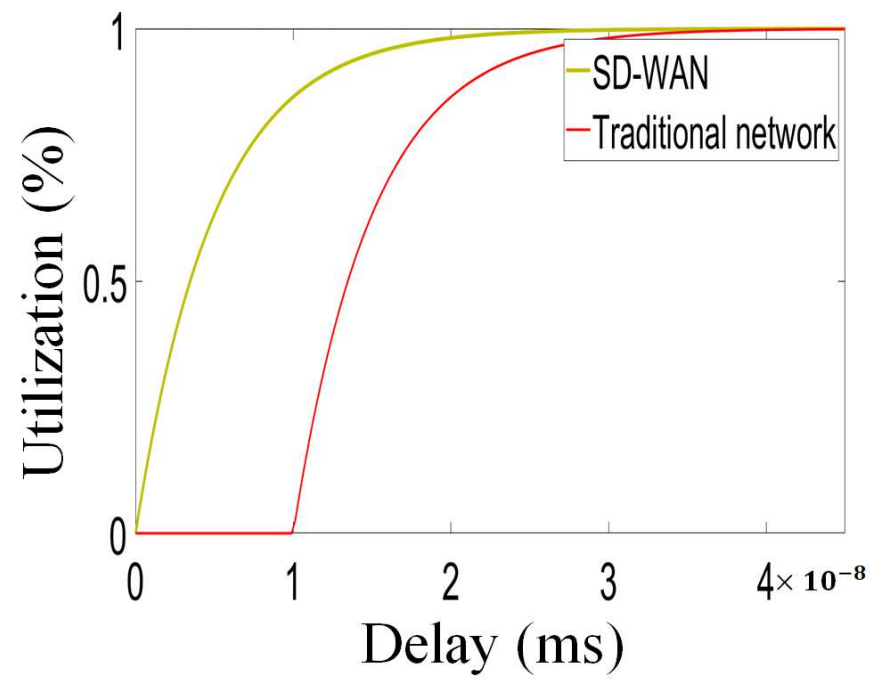

Figure 8. Difference between Delay in SD-WAN and traditional networks

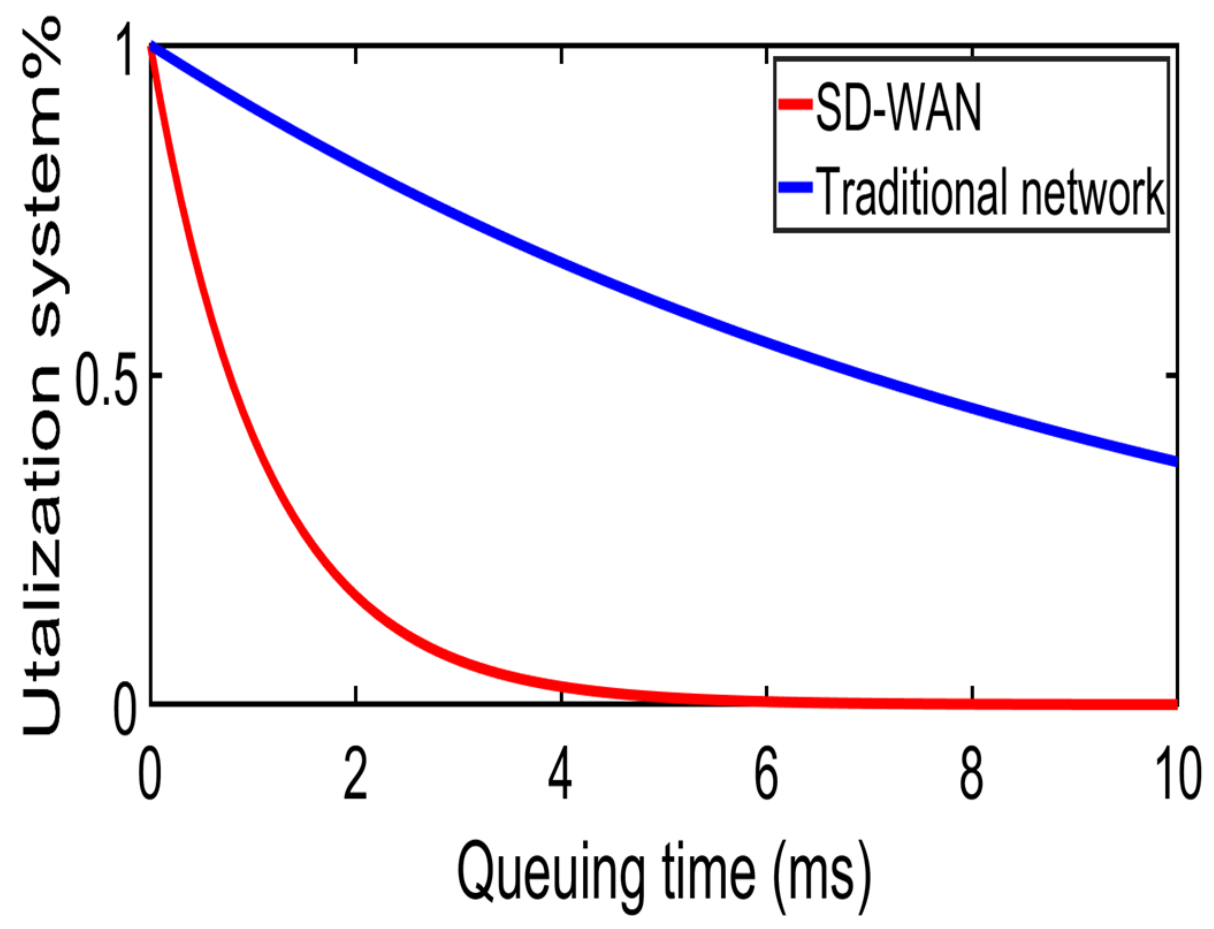

Figure 9. The relationship between utilization and Queuing time in SD-WAN network

model, as well as studying the factors affecting the arrival of the packet and its average waiting time in queuing before entering service, as well as calculating the average time of the whole system, thus reducing message delay.

\section{References}

1. De, D., Mukherjee, A., Das, S. K. \& Dey, N. Nature inspired computing for wireless sensor networks (Springer, 2020).

2. Troia, S., Zorello, L. M. M., Maralit, A. J. \& Maier, G. Sd-wan: an open-source implementation for enterprise networking services. In 2020 22nd International Conference on Transparent Optical Networks (ICTON), 1-4, DOI: 10.1109/ ICTON51198.2020.9203058 (IEEE, 2020). 


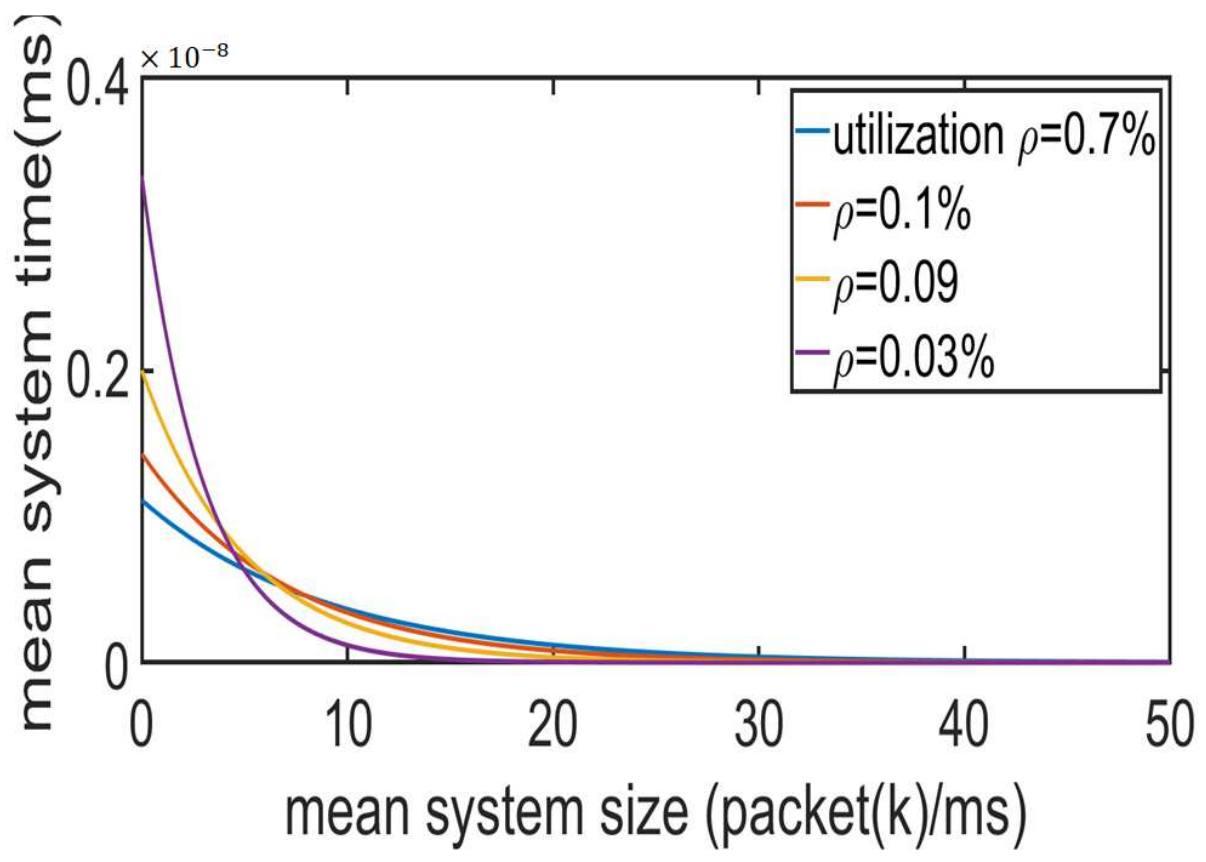

Figure 10. The relationship between Waiting time and mean system size in SD-WAN network

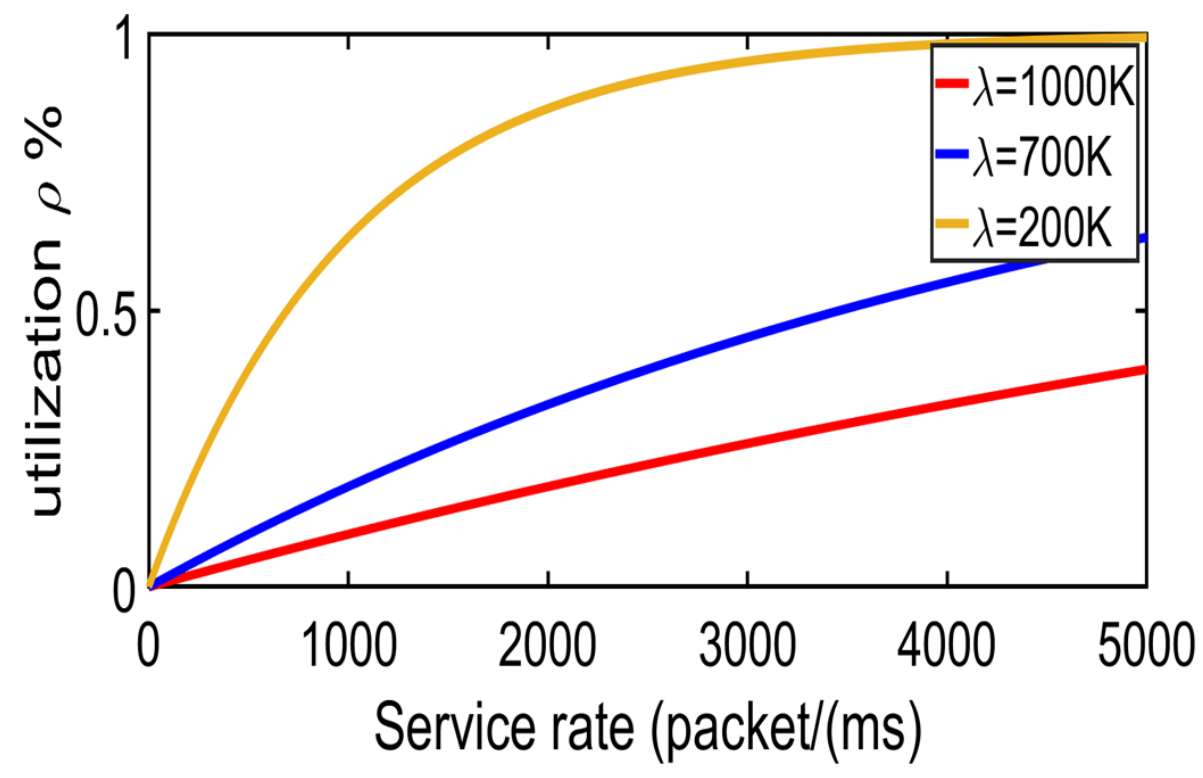

Figure 11. The relationship between utilization and service rate in SD-WAN network

3. Spezio, A. E. Electronic warfare systems. IEEE Transactions on Microw. Theory Tech. 50, 633-644, DOI: 10.1109/22. 989948 (2002).

4. Tang, Y. et al. A survey on the $5 \mathrm{~g}$ network and its impact on agriculture: Challenges and opportunities. Comput. Electron. Agric. 180, 105895, DOI: https://doi.org/10.1016/j.compag.2020.105895 (2021).

5. Yang, Z., Cui, Y., Li, B., Liu, Y. \& Xu, Y. Software-defined wide area network (sd-wan): Architecture, advances and opportunities. In 2019 28th International Conference on Computer Communication and Networks (ICCCN), 1-9 (IEEE, 2019).

6. Nweke, L. O. \& Wolthusen, S. D. Modelling adversarial flow in software-defined industrial control networks using a 


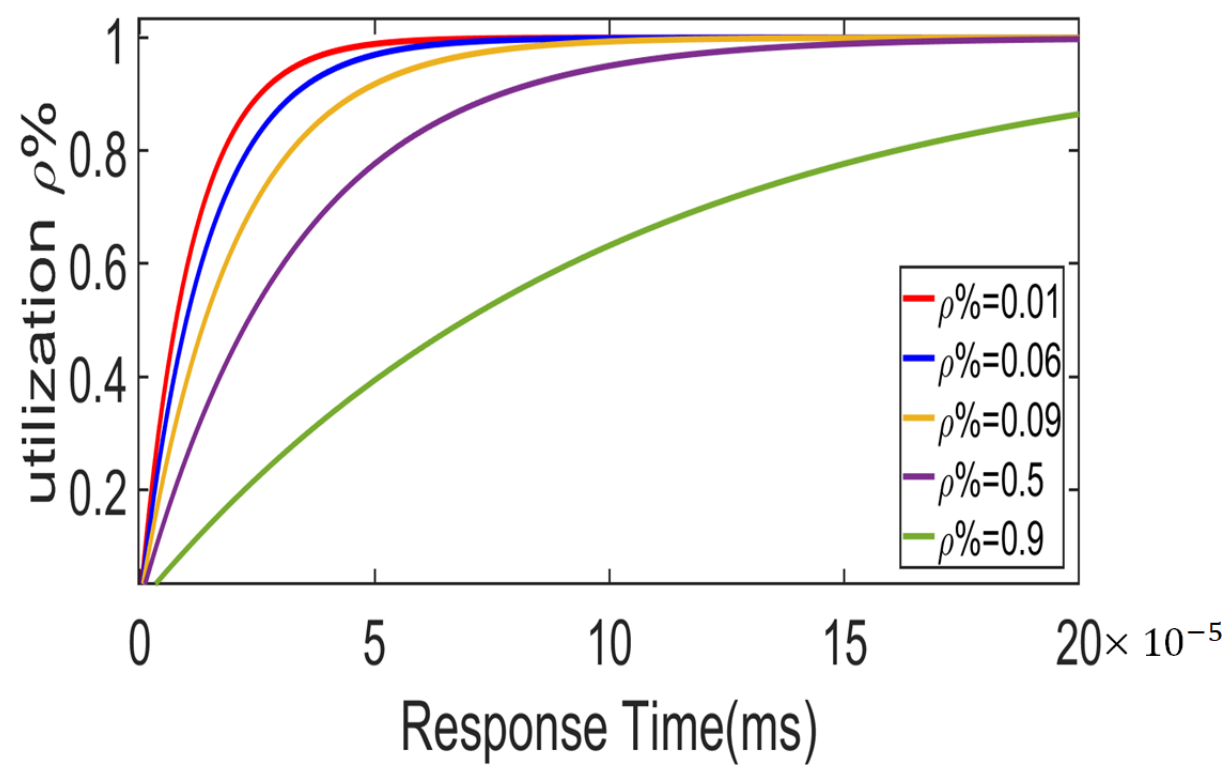

Figure 12. The relationship between utilization and Response time in SD-WAN network

queueing network model. In 2020 IEEE Conference on Communications and Network Security (CNS), 1-6 (IEEE, 2020).

7. Muhizi, S. et al. Analysis and performance evaluation of sdn queue model. In International Conference on Wired/Wireless Internet Communication, 26-37 (Springer, 2017).

8. Goto, Y., Ng, B., Seah, W. K. \& Takahashi, Y. Queueing analysis of software defined network with realistic openflow-based switch model. Comput. Networks 164, 106892 (2019).

9. Durner, R., Blenk, A. \& Kellerer, W. Performance study of dynamic qos management for openflow-enabled sdn switches. In 2015 IEEE 23rd International Symposium on Quality of Service (IWQoS), 177-182 (IEEE, 2015).

10. Zhao, J., Hu, Z., Xiong, B., Yang, L. \& Li, K. Modeling and optimization of packet forwarding performance in softwaredefined wan. Futur. Gener. Comput. Syst. 106, 412-425, DOI: https://doi.org/10.1016/j.future.2019.12.010 (2020).

11. Yang, Z., Cui, Y., Li, B., Liu, Y. \& Xu, Y. Software-defined wide area network (sd-wan): Architecture, advances and opportunities. In 2019 28th International Conference on Computer Communication and Networks (ICCCN), 1-9 (IEEE, 2019).

12. Neuts, M. F. \& Pagano, M. E. Generating random variates from a distribution of phase type. Tech. Rep., Institute of Electrical and Electronics Engineers (IEEE) (1981).

13. Luh, H. \& Xu, Z.-Z. Ph/ph/1 queueing models in mathematica for performance evaluation. Int. J. Oper. Res. 2, 81-88 (2005).

14. Stewart, W. J. Queues with phase-type laws: Neuts' matrix-geometric method. In Probability, Markov Chains, Queues, and Simulation, 444-474 (Princeton University Press, 2009).

15. Neuts, M. F. Phase-type Probability Distributions, 1132-1134 (Springer US, Boston, MA, 2013).

16. Mor, H.-B. Performance modeling and design of computer systems, vol. 576 (Cambridge University Press, 2013).

17. Harchol-Balter, M. Performance modeling and design of computer systems: queueing theory in action (Cambridge University Press, 2013). 


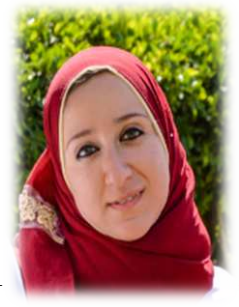

]SAMAA ADEL IBRAHIM HUSSIEN is a researcher at Faculty of Engineering, Mansoura University, Egypt. She Received the B.S.C. degree in Comm. Her current research interests include addressing and combating digital electronic warfare, protecting networks from electronic attacks and electromagnetic bombs, SD-WAN, automation, Cloud Computing, and Internet routing.

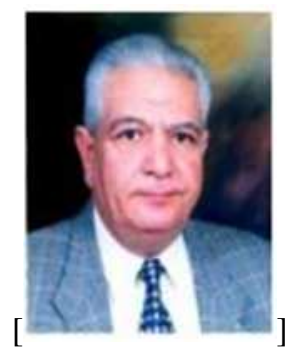

]FAYEZ W. ZAKI is a professor at the Faculty of Engineering, Mansoura University. He received the B. Sc. in Communication Eng. from Menofia University Egypt 1969, M. Sc. Communication Eng. from Helwan University Egypt 1975, and Ph.D. from Liverpool University U.K 1982. Head of Electronics and Communication Engineering Department Faculty of Engineering, Mansoura University from 2002 till 2005. He supervised several MSc and Ph.D. thesis. He has published several papers in refereed journals and international conferences. He is now a member of the professorship promotion committee in Egypt.

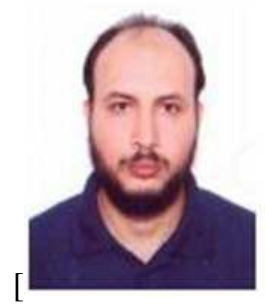

]MOHAMMED M. ASHOUR is an assistant professor at the faculty of engineering Mansoura University, Egypt. He received B.Sc. and M.Sc. from Mansoura University Egypt. He received the Ph.D. degree from Mansoura University, Egypt 2005. Worked as Lecturer Assistant at Mansoura University, Egypt from 1997, from 2005, an Assistant Professor. 